pathogenesis and correlates of fatal outcome. Clin Infect Dis. 2012;54:527-33. http://dx.doi.org/10.1093/cid/cir804

10. Tamura K, Stecher G, Peterson D, Filipski A, Kumar S. MEGA6: Molecular Evolutionary Genetics Analysis version 6.0. Mol Biol Evol. 2013;30:2725-9. http://dx.doi.org/10.1093/molbev/ mst197

Address for correspondence: Keun Hwa Lee, Department of Microbiology and Immunology, Jeju National University College of Medicine, 15, Aran 13-gil, Jeju 63241, South Korea; email: yomust7@jejunu.ac.kr

\section{Spotted Fever Group Rickettsiae in Inner Mongolia, China, 2015-2016}

\author{
Gaowa, Wulantuya, Xuhong Yin, Shengchun Guo, \\ Chunlian Ding, Minzhi Cao, Hiroki Kawabata, \\ Kozue Sato, Shuji Ando, Hiromi Fujita, \\ Fumihiko Kawamori, Hongru Su, \\ Masahiko Shimada, Yuko Shimamura, \\ Shuichi Masuda, Norio Ohashi
}

\begin{abstract}
Author affiliations: College of Hetao, Bayan Nur City, Inner Mongolia, China (Gaowa, Wulantuya, X. Yin, S. Guo, C. Ding); Bayan Nur Centers for Disease Control and Prevention, Bayan Nur City (M. Cao); National Institute of Infectious Diseases, Shinjuku-ku, Tokyo, Japan (H. Kawabata, K. Sato, S. Ando); Mahara Institute of Medical Acarology, Anan City, Tokushima, Japan (H. Fujita); University of Shizuoka, Shizuoka City, Japan (F. Kawamori, H. Su, M. Shimada, Y. Shimamura, S. Masuda, N. Ohashi)
\end{abstract}

DOI: https://doi.org/10.3201/eid2411.162094

We found Rickettsia raoultii infection in 6/261 brucellosisnegative patients with fever of unknown origin in brucellosis-endemic Inner Mongolia, China. We further identified Hyalomma asiaticum ticks associated with $R$. raoultii, $H$. marginatum ticks associated with $R$. aeschlimannii, and Dermacentor nuttalli ticks associated with both rickettsiae species in the autonomous region.

Qpotted fever group rickettsiae (SFGR) are vector$\checkmark$ borne pathogens. In China, 5 SFGR genotypes have been identified as causative agents of human rickettsiosis: $R$. heilongjiangensis, $R$. sibirica subsp. sibirica BJ-90,
Candidatus Rickettsia tarasevichiae, $R$. raoultii, and Rickettsia sp. XY99 (1-4).

Brucellosis, a zoonotic disease, is highly endemic to Inner Mongolia, China, and is increasing in workers in agriculture or animal husbandry (5). However, some agriculture workers with brucellosis-like symptoms, including general malaise and fever, were seronegative for Brucella spp. We suspected that fever of unknown origin among brucellosis-seronegative patients might be caused by tickborne pathogens. We identified 6 cases of human $R$. raoultii infections in brucellosis-seronegative patients in western Inner Mongolia, and we investigated exposure to ticks infected with SFGR.

During 2015-2016, we obtained 261 blood samples from brucellosis-seronegative patients with fever of unknown origin in Bayan Nur Centers for Disease Control and Prevention (Bayan Nur City, Inner Mongolia, China). The review board of the Department of Medicine at College of Hetao (Bayan Nur City) approved the study. We extracted DNA from each blood sample using the DNeasy Mini Kit (QIAGEN, Hilden, Germany) and conducted PCR targeting SFGR gltA (6). The PCR primers used, gltA-Fc (5'-CGAACTTACCGCTATTAGAATG-3') and gltA-Rc (5'-CTTTAAGAGCGATAGCTTCAAG-3'), were described previously (4). We designed the primers $16 \mathrm{~S}$ rDNA R-2F (5'-GAAGATTCTCTTTCGGTTTCGC-3'), 16S rDNA R-2R (5'-GTCTTGCTTCCCTCTGTAAAC-3'), rompA-Fb (5'-GGTGCGAATATAGACCCTGA-3'), and rompA-Ra (5'-TTAGCTTCAGAGCCTGACCA-3') for this study and deposited the sequences obtained of glt $A$, ompA, and $16 \mathrm{~S}$ rDNA into GenBank (accession nos. MH267733-47). We used genomic DNA extracted from L929 cells infected with Rickettsia sp. LON-13 (gltA: AB516964) as a positive control.

We detected gltA amplicons from 6/261 (2.3\%) blood samples (Table). All 6 patients had strong malaise and mild fever of $36.8^{\circ} \mathrm{C}-37.3^{\circ} \mathrm{C}$ but no rash. Five of these patients also had arthralgia and vomiting.

Sequence and phylogenetic analysis showed that the sequences of 6 nearly full-length $(1.1 \mathrm{~kb}) \mathrm{glt} A$ amplicons with were identical to each other and to $R$. raoultii gltA (GenBank accession no. DQ365803). We further analyzed ompA and $16 \mathrm{~S}$ rDNA in gltA-positive samples. All 6 samples were PCR positive for both genes; 552-bp sequences of the amplicons were identical to sequences of $R$. raoultii ompA (GenBank accession no. AH015610), and 389-bp sequences of the amplicons were identical to sequences of $R$. raoultii $16 \mathrm{~S}$ rDNA (GenBank accession no. EU036982). PCR results were negative for the genes Anaplasma phagocytophilum p44/msp2, Ehrlichia chaffeensis p28/omp-1, and Borrelia spp. flaB. An indirect immunofluorescence assay showed that $\operatorname{IgM}$ and IgG titers against $R$. japonica were $40-80$ for IgM in 3 patients and 160 for $\mathrm{IgG}$ in 2 patients. 
Table. PCR survey of SFGR infections in patients and ticks, Inner Mongolia, China, 2015-2016*

\begin{tabular}{lcccc}
\hline & & \multicolumn{3}{c}{ No. $(\%)$ gltA positive for SFGR } \\
\cline { 3 - 5 } Patient type or tick species & No. tested & Rickettsia raoultii & $R$. aeschlimannii & Total \\
\hline Brucellosis-seronegative patients & 261 & $6(2.3)$ & 0 & $6(2.3)$ \\
\hline Ticks & & & 0 & $118(15.4)$ \\
$\quad$ Hyalomma asiaticum & 766 & 0 & $160(80.8)$ & $160(80.8)$ \\
$\quad$ Hyalomma marginatum & 198 & $830(58.5)$ & $158(11.1)$ & $988(69.7) \dagger$ \\
Dermacentor nuttalli & 1,418 & 0 & 0 & 0 \\
$\quad$ Rhipicephalus turanicus & 76 & $948(38.6)$ & $318(12.9)$ & $1,266(51.5)$ \\
$\quad$ Total ticks & 2,458 & &
\end{tabular}

${ }^{*}$ SFGR, spotted fever group rickettsiae.

†We did not detect dual infection with $R$. raoultii and $R$. aeschlimannii in $D$. nuttalli ticks in this study.

To assess patients' risk of infection with SFGR by tick exposure, we collected 2,458 ticks morphologically identified as Hyalomma marginatum $(\mathrm{n}=198), \mathrm{H}$. asiaticum $(\mathrm{n}=766)$, Dermacentor nuttalli $(\mathrm{n}=1,418)$, and Rhipicephalus turanicus $(\mathrm{n}=76)$ from livestock and pet animals including sheep, cattle, camels, and dogs in western Inner Mongolia during 2015-2016 (online Technical Appendix Figure 1, https://wwwnc.cdc.gov/EID/ article/24/11/16-2094-Techapp1.pdf). We collected unattached ticks within animal hair, but not attached ticks. We prepared DNA extracted from salivary glands of each tick and conducted PCR screening by rickettsial gltA detection as described. We detected gltA in 1,266 (51.5\%) of the total 2,458 ticks.

We classified the amplicons into 2 groups by restriction fragment-length polymorphism using $A l u \mathrm{I}$ and $R s a \mathrm{I}$, and we sequenced 25-45 representative amplicons in each group. On the basis of this analysis, we found that the sequences from the 2 groups were either identical to that of R. raoultii (GenBank accession no. DQ365803) or to that of $R$. aeschlimannii (GenBank accession no. HM050276) (Table; online Technical Appendix Figure 2). We detected R. raoultii DNA in $H$. asiaticum $(118 / 766,15.4 \%)$ and $D$. nuttalli $(830 / 1,418,58.5 \%)$ ticks and $R$. aeschlimannii DNA from $H$. marginatum $(160 / 198,80.8 \%)$ and D. nuttalli $(158 / 1,418,11.1 \%)$ ticks. We did not detect rickettsial DNA in $R$. turanicus ticks $(0 / 76,0 \%)$.

Recently, human cases of $R$. raoultii infection have been reported in China, including northeastern Inner Mongolia $(1,4)$. Potential vectors for $R$. raoultii are Dermacentor spp. ticks in Europe, Turkey, and northern Asia and Haemaphysalis spp. and Amblyomma sp. ticks in southern Asia $(7,8)$. Other studies have identified Hyalomma spp., Rhipicephalus spp., and Amblyomma sp. ticks as potential vectors for $R$. aeschlimannii $(7,8)$; human cases of $R$. aeschlimannii infection have been reported in Italy and Morocco (7,9). We detected $R$. raoultii in $H$. asiaticum as well as $D$. nuttalli ticks, but in Mongolia, $R$. raoultii has been detected only in D. nuttalli ticks, and not $H$. asiaticum ticks (10). We identified D. nuttalli ticks as another potential vector for $R$. aeschlimannii. Our work contributes to the knowledge of the epidemiology, clinical characteristics, and known tick vectors associated with $R$. raoultii and $R$. aeschlimannii.

\section{Acknowledgments}

We thank Asaka Ikegaya for providing Rickettsia japonica antigen slides.

This work was supported by grants from the National Natural Science Foundation of China (nos. 31660032 and 31660044 ); Natural Science Foundation of Inner Mongolia (2015BS0331); Bayan Nur Science and Technology Project from Bayan Nur Bureau for Science and Technology; Inner Mongolia Higher Education Science and Technology Project (NJZY261); and Startup Fund for Talented Scholar in College of Hetao (to Gaowa). The research was partially supported by the Research Program on Emerging and Re-emerging Infectious Diseases from Japan Agency for Medical Research and Development (AMED) to N.O., H.K., and S.A.

\section{About the Author}

Dr. Gaowa is an associate professor in Inner Mongolia Key Laboratory of Tick-Borne Zoonosis, Department of Medicine, College of Hetao, Bayan Nur, Inner Mongolia, China. Her primary research interests are molecular biology, ecology, and epidemiology of zoonotic parasites, especially tickborne pathogens.

\section{References}

1. Jia N, Zheng YC, Ma L, Huo QB, Ni XB, Jiang BG, et al. Human infections with Rickettsia raoultii, China. Emerg Infect Dis. 2014;20:866-8. http://dx.doi.org/10.3201/eid2005.130995

2. Fang LQ, Liu K, Li XL, Liang S, Yang Y, Yao HW, et al. Emerging tick-borne infections in mainland China: an increasing public health threat. Lancet Infect Dis. 2015;15:1467-79. http://dx.doi.org/10.1016/S1473-3099(15)00177-2

3. Li H, Cui XM, Cui N, Yang ZD, Hu JG, Fan YD, et al. Human infection with novel spotted fever group Rickettsia genotype, China, 2015. Emerg Infect Dis. 2016;22:2153-6. http://dx.doi.org/10.3201/eid2212.160962

4. Li H, Zhang PH, Huang Y, Du J, Cui N, Yang ZD, et al. Isolation and identification of Rickettsia raoultii in human cases: a surveillance study in 3 medical centers in China. Clin Infect Dis. 2018;66:1109-15. http://dx.doi.org/10.1093/cid/cix917

5. Li MT, Sun GQ, Zhang WY, Jin Z. Model-based evaluation of strategies to control brucellosis in China. Int J Environ Res Public Health. 2017;14:295. http://dx.doi.org/10.3390/ijerph14030295

6. Gaowa, Ohashi N, Aochi M, Wuritu D, Wu, Yoshikawa Y, et al. Rickettsiae in ticks, Japan, 2007-2011. Emerg Infect Dis. 2013;19:338-40. http://dx.doi.org/10.3201/eid1902.120856

7. Parola P, Paddock CD, Socolovschi C, Labruna MB, Mediannikov O, Kernif T, et al. Update on tick-borne rickettsioses around the world: a geographic approach. Clin 
Microbiol Rev. 2013;26:657-702. 1 http://dx.doi.org/10.1128/ CMR.00032-13

8. Karasartova D, Gureser AS, Gokce T, Celebi B, Yapar D, Keskin A, et al. Bacterial and protozoal pathogens found in ticks collected from humans in Corum province of Turkey. PLoS Negl Trop Dis. 2018;12:e006395. http://dx.doi.org/10.1371/journal.pntd.0006395

9. Tosoni A, Mirijello A, Ciervo A, Mancini F, Rezza G, Damiano F, et al.; Internal Medicine Sepsis Study Group. Human Rickettsia aeschlimannii infection: first case with acute hepatitis and review of the literature. Eur Rev Med Pharmacol Sci. 2016;20:2630-3.

10. Boldbaatar B, Jiang RR, von Fricken ME, Lkhagvatseren S, Nymadawa P, Baigalmaa B, et al. Distribution and molecular characteristics of rickettsiae found in ticks across Central Mongolia. Parasit Vectors. 2017;10:61. http://dx.doi.org/10.1186/ s13071-017-1981-3

Address for correspondence: Norio Ohashi, University of Shizuoka, Laboratory of Microbiology, Department of Food Science and Biotechnology, School of Food and Nutritional Sciences, Graduate School of Integrated Pharmaceutical and Nutritional Sciences, 52-1 Yada, Suruga-ku, Shizuoka 422-8526, Japan; email: ohashi@u-shizuoka-ken.ac.jp

\section{Japanese Spotted Fever in Eastern China, 2013}

\section{Jiabin Li, ${ }^{1}$ Wen Hu, ${ }^{1}$ Ting Wu, Hong-Bin Li, Wanfu Hu, Yong Sun, Zhen Chen, Yonglin Shi, Jia Zong, Adams Latif, Linding Wang, Li Yu, Xue-Jie Yu, Bo-Yu Liu, Yan Liu}

\begin{abstract}
Author affiliations: The First Affiliated Hospital of Anhui Medical University, Hefei, China (J. Li, T. Wu, H.-B. Li); The First Affiliated Hospital of the University of Science and Technology of China, Hefei (Wen Hu); Anhui Center for Disease Control and Prevention, Hefei (Wanfu Hu, Y. Sun, Y. Shi); Anhui Medical University, Hefei (Z. Chen, J. Zong, A. Latif, L. Wang, L. Yu, B.-Y. Liu, Y. Liu); Wuhan University School of Health Sciences, Wuhan, China (X.-J. Yu)
\end{abstract}

DOI: https://doi.org/10.3201/eid2411.170264

We isolated Rickettsia japonica from a febrile patient in Lu'an City, China, in 2013. Subsequently, we found an $R$. japonica seroprevalence of $54.8 \%(494 / 902)$ in the rural population of Anhui Province and an R. japonica prevalence in Haemaphysalis longicornis ticks of $0.5 \%$ (5/935). $R$. japonica and its tick vector exist in China.

${ }^{1}$ These authors contributed equally to this article.
Q potted fever group rickettsiae are tickborne, obliga$\checkmark$ tory intracellular, gram-negative bacteria with a worldwide distribution. However, the distribution of each species of spotted fever group rickettsiae is limited to geographic areas by their specific tick vectors. Japanese spotted fever is a severe rickettsiosis caused by Rickettsia japonica bacterium $(1,2)$, which has been present in Japan since 1984 and isolated from patients in other countries of Asia (e.g., South Korea, the Philippines, and Thailand) over the past decade $(3,4)$. In this study, we present information on an $R$. japonica isolate acquired from a febrile patient and $R$. japonica seroprevalence in Anhui Province in eastern China.

On August 7, 2013, a 61-year-old man from Shucheng County, Lu'an City, China, in the Dabie Mountain area of Anhui Province (online Technical Appendix Figure 1, https:// wwwnc.cdc.gov/EID/article/24/11/17-0264-Techapp1. pdf) with fever and headache for 1 week was admitted into Shucheng County People's Hospital. The patient reported several tick bites 10 days before the onset of his illness. At admission, the patient was conscious and had fever $\left(39.0^{\circ} \mathrm{C}\right)$; he did not have jaundice, and no bleeding was found on his skin or mucosal membranes. A papular rash with papules $0.1-0.5 \mathrm{~cm}$ in diameter was noted all over his body (online Technical Appendix Figure 2). Blood cell counts showed the patient had leukocytosis $\left(10.34 \times 10^{9}\right.$ cells/L), increased neutrophils $(87.5 \%)$, and a platelet count within reference range $\left(130 \times 10^{9} / \mathrm{L}\right)$. Blood chemistry testing revealed a urea nitrogen concentration of $9.12 \mathrm{mmol} / \mathrm{L}$ (reference range 2.9-8.2 $\mathrm{mmol} / \mathrm{L})$, creatinine of $0.758 \mathrm{mg} / \mathrm{dL}(67 \mu \mathrm{mol} / \mathrm{L}$, reference range $53-106 \mu \mathrm{mol} / \mathrm{L}$ ), C-reactive protein of $77.5 \mathrm{nmol} / \mathrm{L}$ (reference range $0.76-28.5 \mathrm{nmol} / \mathrm{L}$ ), and an erythrocyte sedimentation rate of $22 \mathrm{~mm} / \mathrm{h}$ (reference range $0-20 \mathrm{~mm} / \mathrm{h}$ ). A urine test showed a procalcitonin concentration of $0.806 \mathrm{ng} /$ $\mathrm{mL}$ (reference range $\leq 0.15 \mathrm{ng} / \mathrm{mL}$ ) and an interleukin 6 concentration of $52 \mathrm{pg} / \mathrm{mL}$ (reference range $\leq 1.8 \mathrm{pg} / \mathrm{mL}$ ). The patient had rough lung breath sounds, and computed tomography showed inflammatory infiltrates in the middle right lung and lower left lung lobe, bullae on the upper left lung lobe, and emphysematous changes. The patient was suspected to have a rickettsial infection and was given minocycline and meropenem on the day of his admission. Two days later, on August 9,2013 , the patient's fever subsided $\left(36.2^{\circ} \mathrm{C}\right)$, and he was discharged.

A blood sample taken from the patient 1 day after admission was inoculated onto THP-1 and Vero E6 cells; after 10 days, cytopathic effect was visible by light microscopy with only the THP-1 cells. Diff-Quick (Thermo Fisher Scientific, Kalamazoo, MI, USA)-stained smears of THP-1 cells showed Rickettsia-like bacilli in the cytoplasm. Electron microscopy showed the bacilli localized to the cytoplasm and nucleus and had the typical ultrastructure of Rickettsia bacteria. This species was highly pleomorphic 